\title{
Amiloidosis hepática como causa de colestasis severa intrahepática
}

\author{
J. C. GAVILÁN, F. J. BERMÚDEZ, A. MÁRQUEZ1' J. J. SÁNCHEZ-CARRILLO', \\ P. GONZÁLEZ-SANTOS
}

Servicio de Medicina Interna $y^{1}$ Anatomía Patológica. Hospital Universitario Virgen de la Victoria. Málaga

\author{
PRIMARY AMYLOIDOSIS AS A CAUSE OF SEVERE INTRAHEPATIC \\ CHOLESTASIS
}

\begin{abstract}
RESUMEN
El hígado suele verse afectado en la amiloidosis sistémica; sin embargo, la hiperbilirrubinemia y los signos de fallo hepático son manifestaciones muy poco comunes. Una elevación ligera de las cifras de fosfatasa alcalina y, menos frecuentemente, la existencia de hepatomegalia son los hallazgos más habituales. Generalmente, los pacientes no refieren manifestaciones clínicas relacionadas con la afectación hepática; las manifestaciones clínicas y el pronóstico a largo plazo dependen de la existencia de afectación renal y cardíaca. Comunicamos un caso de amiloidosis primaria con una presentación clínica poco usual, en una mujer de 65 años de edad, que fue ingresada por un cuadro de ictericia, ascitis y fallo hepático agudo atribuído a hepatitis inducida por fármacos.
\end{abstract}

PALABRAS CLAVE: Amiloidosis AL. Amiloidosis hepática. Ictericia. Fallo hepático agudo.

\begin{abstract}
The liver is frequently involved by amyloidosis, but hyperbilirubine mia and liver failure are uncommon features. A mild elevation of the serum alkaline phosphatase value and, less frequently, hepatomegaly are the most common findings. Usually the patients have no symptoms rela ted with the liver involvement; the clinical manifestation and the long term prognosis depends on the renal and cardiac disease. We report an unusual clinical presentation of primary amyloidosis in a previously asymptomatic 65 years old woman who was admitted to the hospital because of ictericia and ascitis mimicking a drug induced acute hepatic failure.
\end{abstract}

KEY WORDS: AL amyloidosis. Hepatic amyloidosis. Jaundice. Acute hepatic failure.

Gavilán JC, Bermúdez FJ, Márquez, A, Sánchez-Carrillo JJ, González-Santos P. Amiloidosis hepática como causa de colestasis severa intra hepática. An Med Interna (Madrid) 2003; 20: 25-27.

\section{INTRODUCCIÓN}

La amiloidosis sistémica es un proceso patológico que se produce como consecuencia del depósito de un material proteináceo amorfo en el espacio extracelular de muchos órganos y tejidos (amiloide). Existen varias formas de material amiloide con características propias tanto desde el punto de vista clínico como bioquímico, pero todas ellas comparten una estructura secundaria idéntica (plegamiento beta), que les confiere unas propiedades tintoriales peculiares. Desde el punto de vista fisiopatológico, los depósitos masivos de material amiloide en los tejidos lleva a la desorganización de la arquitectura normal de los mismos y a pérdida de parte de los elementos estructurales normales que lo componen.

La clasificación de la amiloidosis sistémica se basa en la naturaleza bioquímica del material fibrilar protéico precursor del cuadro. En la nomenclatura de las distintas formas de amiloide se usa una primera letra A, seguida de una segunda inicial que designa el origen de la proteína precursora: así, en la amiloidosis primaria el precursor protéico esta formado por cadenas ligeras de lnmunoglobulinas (AL); en las formas secundarias, el precursor lo constituye la proteína sérica A (AA); la transtirretina (prealbúmina) es la proteína precursora en algunas formas familiares de amiloidosis y en la amiloidosis sistémica senil (ATTR), mientras que en las formas asociadas a diálisis el precursor lo constituye una beta- 2 microglobulina $(\mathrm{Ab} 2 \mathrm{~m})$. Los depósitos amiloideos contienen un componente no fibrilar común a todos ellos, que es la pentraxina o amiloide sérico $P$.

La amiloidosis por depósitos de cadenas ligeras (AL) es la forma más común de amiloidosis sistémica (1). El material fibrilar de la amiloidosis AL está compuesto por restos de aminoácidos de la región variable de una cadena ligera monoclonal. Desde el punto de vista clínico, en la amiloidosis AL diferenciamos dos subgrupos: a) amiloidosis AL asociada a mieloma múltiple (constituye el $20 \%$ ). En esta forma, al igual que ocurre en el mieloma, la cadena ligera más frecuente es Kappa, y b) amiloidosis AL primaria -no asociada a mielo-

Trabajo aceptado: 17 de octubre de 2001 
ma- $(80 \%$ de los casos $)$, en la que la cadena monoclonal predominante es tipo lambda (2:1). En ambos casos, el exceso de cadenas monoclonales, kappa o lambda -o de fragmentos de las mismas- son procesados de forma anormal por las enzimas de los macrófagos, para dar lugar a cadenas ligeras parcialmente degradadas precursoras de la amiloidosis AL.

La afectación renal y/o cardiaca constituye el principal evento clínico en la amiloidosis sistémica, determinando la afectación de estos órganos, frecuentemente, el pronóstico final del paciente. En el hígado es común la presencia de depósito amiloideo; sin embargo, es muy infrecuente que el paciente presente síntomas relacionados con ello. A continuación describimos el caso de una paciente cuya forma de debut clínico fue un fallo hepático agudo.

\section{CASO APORTADO}

Mujer de 65 años de edad, previamente asintomática, que fue ingresada en nuestro centro por ictericia progresiva, edemas y ascitis. La paciente se había sentido bien hasta tres semanas antes del ingreso, cuando, por prescripción de su médico, había iniciado un tratamiento hipolipemiante (pravastatina: $20 \mathrm{mg} / \mathrm{d}$ ). Pocos días después, comenzó a notar molestias abdominales difusas, coluria y anorexia progresiva. La paciente atribuyó sus síntomas a la medicación prescrita, y la suspendió. En su historia personal no había antecedentes de enfermedad hepática previa, abuso de alcohol o tóxicos ni factores de riesgo de exposición a virus de hepatitis, excepto una transfusión sanguínea que se le había realizado 20 años antes.

El examen físico al ingreso reflejaba la existencia de ictericia, edemas periféricos y ascitis, sin flapping ni signos de encefalopatía. En la auscultación se apreciaba un soplo sistólico eyectivo en borde paraesternal izquierdo y la exploración pulmonar fue normal.

Pruebas complementarias. El estudio de sangre periférica fue normal. Los principales resultados de los estudios bioquímicos y serológicos llevados a cabo se expresan en la Tabla I. La inmunoelectroforesis sérica no demostró la existencia de pico monoclonal. Los estudios virológicos indicaron la existencia de infección por el virus de la hepatitis B ( $\mathrm{HbsAg}+)$, sin datos de replicación viral (HbeAg y DNA-VHB -), y por el virus de la hepatitis C (Elisa-antiVHC +). La ecografía abdominal mostraba un hígado aumentado de tamaño, con

TABLA I

PRINCIPALES RESULTADOS BIO Q UÍM ICOS Y SERO LÓ GICOS

\begin{tabular}{ll}
\hline Parámetros & Valores \\
\hline Creatinina & $1,7 \mathrm{mg} / \mathrm{dl}$ \\
BUN & $92 \mathrm{mg} / \mathrm{dl}$ \\
AST & $162 \mathrm{U} / \mathrm{l}$ \\
ALT & $73 \mathrm{u} / \mathrm{l}$ \\
GGT & $820 \mathrm{U} / \mathrm{l}$ \\
F. alcalina & $1117 \mathrm{U} / \mathrm{I}$ \\
Bilirrubina total & $9,4 \mathrm{mg} / \mathrm{dl}$ \\
Bilirrubina directa & $9,0 \mathrm{mg} / \mathrm{dl}$ \\
Colesterol & $587 \mathrm{mg} / \mathrm{dl}$ \\
Albúmina sérica & $25 \mathrm{~g} / \mathrm{l}$ \\
Proteinuria en orina de $24 \mathrm{~h}$. & $2,98 \mathrm{~g} / 24 \mathrm{~h}$ \\
HbsAg & Positivo \\
HbeAg & Negativo \\
AntiHBc Ig M & Negativo \\
AntiVHC (Elisa) & Positivo \\
RNA-VHC (PCR) & Negativo \\
\hline
\end{tabular}

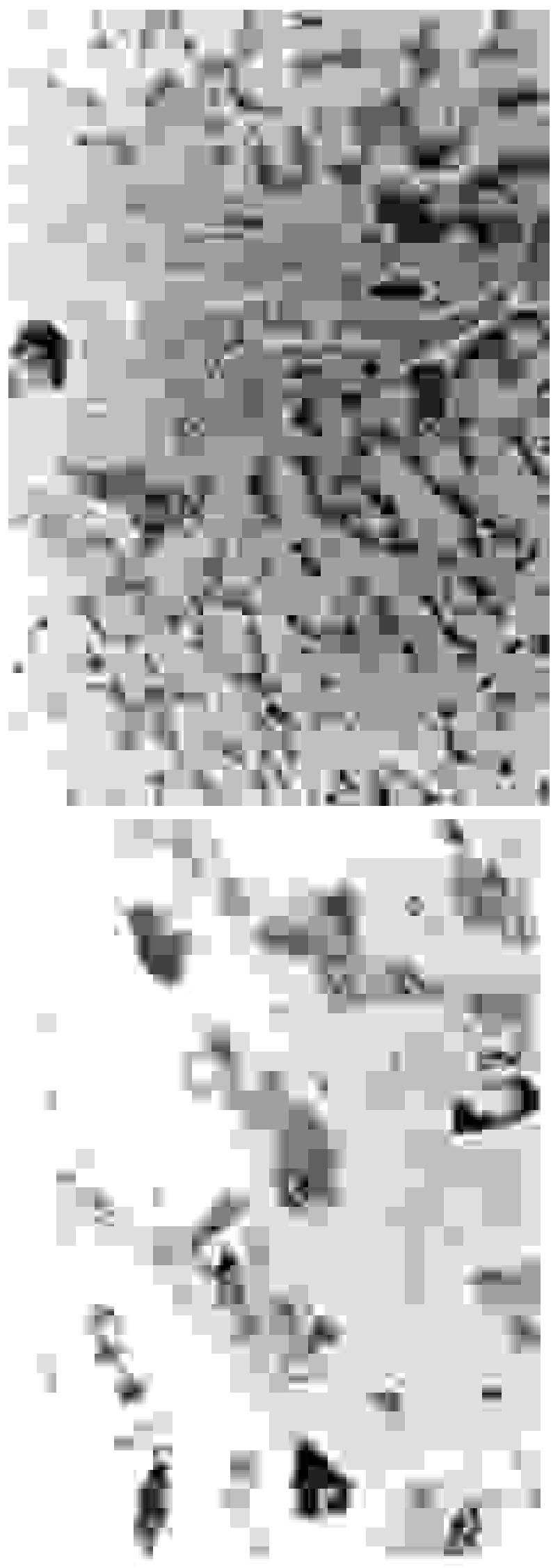

Fig. 1. Imagen microscópica del hígado mostrando un infiltrado severo de los sinusoides hepáticos por amiloide, con desplazamiento de las trabéculas hepáticas. 
ecoestructura homogénea y abundante ascitis. El colédoco y la vía biliar intrahepática no estaban dilatadas. La porta y las venas suprahepáticas estaban permeables y de tamaño normal.

En los 10 días siguientes al ingreso se produjo un deterioro en el estado general de la paciente, con aparición de signos de encefalopatía progresiva y disminución del nivel de conciencia. La paciente falleció por insuficiencia hepatocelular dos semanas después del ingreso. Se llevó a cabo un estudio necrópsico, donde se evidenció la existencia de una hígado aumentado de tamaño, de aspecto no cirrótico. El estudio microscópico demostró la existencia de un infiltrado masivo de material proteináceo homogeneo que desplazaba las trabéculas hepáticas (Fig. 1). Usando la tinción de rojo Congo, y bajo una fuente de luz polarizada, se observó que el material producía birrefringencia verde-manzana. El estudio inmunohistoquímico indicaba que el material extracelular descrito correspondía a una amiloidosis AL tipo cadena Kappa. Por el contrario, no se demostró tincción para el core del virus de la hepatitis B en los nucleos de los hepatocitos.

\section{DISCUSIÓN}

Aunque es común la presencia de depósitos hepáticos en la amiloidosis sistémica (70-90\% de las formas AL y AA), la existencia de anomalías funcionales hepáticas suele ser mínima y ocurrir de forma tardía. Los niveles de fosfatasa alcalina pueden estar ligeramente elevados en un cuarto de los pacientes, con una discreta elevación en las cifras de transaminasas hasta en un $30 \%$ de los casos. La elevación de la bilirrubina por encima de $2 \mathrm{mg} / \mathrm{dl}$ es muy infrecuente (1). No obstante, la hiperbilirrubinemia se asocia a mal pronóstico a corto plazo (2). La hipoalbuminemia es más común, y se relaciona directamente con la existencia de síndrome nefrótico que habitualmente aparece tras el depósito de amiloide AL en los glomérulos renales. Aunque está descrita la existencia de hipertensión portal, es un hecho poco frecuente, lo que explica la baja incidencia de sangrado por rotura de varices esofágicas en estos pacientes. La ascitis puede desarrollarse como consecuencia de aumento de la presión sinusoidal por hipertensión

\section{Bibliografía}

1. Kyler RA. Primary systemic amyloidosis: clinical and laboratory features in 474 cases. Semin Hematol 1995; 32 (1): 45-59.

2. Gertz MA, Kyle RA. Hepatic amyloidosis (primary AL inmunoglobulin light chain): the natural history in 80 patiens. Am J Med 1988; 85: 73 80

3. A, Koff RS, Cohen AS. Severe intrahepatic cholestasis in primary amyloidosis: a report of four cases and a review of the literature. Am J Med 1978; 64: 937-946.

4. Baroja Bengoechea A, Inclan Iribar G, Aguirre Urizar J, Ituarte Arizaga J, Irigoyen oyarzabal A. Amiloidosis hepática como una causa infrecuente de ictericia. Med Clin (Barc) 1981; 77 (9): 393-395.

5. Hoffman MS, Stein BE, Davidian MM, Rosenthal WS. Hepatic amyloidosis presenting as severe intrahepatic cholestasis: a case report and review of the literature. Am J Gastroenterol 1988; 83 (7): 783 785

6. Faa G, Van Eyken P, De Vos R, Fevery J, Van Damme B, De Groote J, Desmet VS. Light chain deposition disease of the liver associated with AL- portal y/o fallo cardíaco congestivo, y se ve favorecido por la hipoalbuminemia que frecuentemente coexiste.

En una revisión de la literatura, hemos encontrado sólo unos pocos casos de amiloidosis hepática cuyo debut clínico fue un cuadro similar a un fallo hepático agudo (3-11). La existencia, en el caso que describimos, de datos de infección por el VHB (HbsAg +) y por el VHC (Elisa-antiVHC) fue un elemento de confusión adicional a la hora de establecer el diagnóstico etiológico del fallo hepático agudo. Este hecho se vio acrecentado por el antecedente de toma de un inhibidor de la HMG-CoA reductasa, al que atribuyó la propia paciente el origen de sus molestias iniciales, y del que se ha descrito en la literatura toxicidad hepática aguda (12). Por otro lado, el grave y rápido deterioro clínico que presentó la paciente, junto a la existencia de ascitis y alteración de la coagulación, imposibilitaron la realización de una biopsia hepática. Sin embargo, algunos datos, como la ausencia de marcadores de infección reciente por el virus de la hepatitis B (antiHBc IgM -), la no detección del RNA del virus $\mathrm{C}$ por reacción en cadena de la polimerasa, el corto periodo de latencia desde la toma de la medicación hasta el inicio de la clínica, así como la existencia de proteinuria en rango nefrótico, nos hicieron sospechar la existencia de otro proceso que justificase el cuadro clínico.

Del análisis del presente caso concluimos, que la biopsia hepática, cuando es posible su realización, constituye un instrumento útil en el diagnóstico etiológico de las hepatopatías agudas graves, especialmente si el origen de las mismas es dudoso, o se sospecha relación con patologías que obligan al diagnóstico de exclusión, como pueden ser las hepatitis tóxicas.

\section{AGRADECIMIENTOS}

Agradecemos la realización del estudio inmunohistoquímico a F. Gavilán y J. Fernández, del Servicio de Anatomía Patológica. Hospital Virgen del Rocio, Sevilla. type amyloidosis and severe cholestasis. J Hepatol 1991; 12 (1): 75-82

7. McDonald P, Usbourne C, Playfer JR. A case of intrahepatic cholestasis due to amyloidosis. Int J Clin Pract 1998; 52 (3): 201-202.

8. Peters RA. Primary amyloidosis and severe intrahepatic cholestasis jaundice. Gut 1994; 35: 1322-1325.

9. Conde Martel A; Suarez Ortega S, Redondo E, Guerra C. Ictericia colestásica, infrecuente manifestación inicial de amiloidosis primaria. Med Clin (Barc) 1995; 104 (14): 557.

10. Goenka MK, Bhasin DK, Vasisth RK, Dhawan S. Hepatic amyloidosis presenting with severe intrahepatic cholestasis. J Clin Gastroenterol 1996; 23 (2): 134-136.

11. Mainenti PP. Primary systemic amyloidosis with giant hepatomegaly and a swiftly progressive course. J Clin Gastroenterol 1997; 24 (3): 173-175.

12. Grimbert S, Pessayre D, Degott C, Benhamou JP. Acute hepatitis induced by HMG-CoA reductase inhibitors. Lovastatin. Dig Dis Sci 1994; 34: 2032-2033. 
